\title{
Application status of tamoxifen in endocrine therapy for early breast cancer
}

\author{
QIANCHENG HU*, TING LUO*, XIAORONG ZHONG, PING HE, TINGLUN TIAN and HONG ZHENG \\ Departments of Head and Neck and Mammary Gland Oncology, and Medical Oncology, \\ Cancer Center; State Key Laboratory of Biotherapy, Laboratory of Molecular Diagnosis of Cancer, \\ West China Hospital, Sichuan University, Chengdu, Sichuan 610041, P.R. China
}

Received February 18, 2014; Accepted July 14, 2014

DOI: $10.3892 /$ etm.2015.2437

\begin{abstract}
The aim of the present study was to investigate the application status of tamoxifen as an adjuvant treatment for early-stage breast cancer. Data for patients with early breast cancer were collected from an information management system for breast cancer in a single hospital between 1989 and 2012. The inclusion criteria included no distant metastasis during diagnosis with breast cancer, and a diagnosis of estrogen and/or progesterone receptor-positive breast cancer, or an unclear hormonal receptor status with tamoxifen used as the preferred drug. A total of 2,738 patients were selected, with $99.4 \%$ female and $0.6 \%$ male patients. Within females, $75.7 \%$ were premenopausal when diagnosed with early breast cancer, $24.3 \%$ were postmenopausal, and the median age of menopause was 47 years. The percentage of tamoxifen preference in adjuvant therapy was $97.3 \%$ prior to 2000 , which decreased to $60.4 \%$ in 2011. Tamoxifen preference among premenopausal patients reduced from $97.3 \%$ prior to 2000 to $89.5 \%$ in 2011 , while in postmenopausal patients tamoxifen preference declined from $97.4 \%$ prior to 2000 to $22.7 \%$ in 2011. One-year duration of tamoxifen treatment accounted for $79.4 \%$ of cases, while five-year duration accounted for $24.7 \%$. The rate of one-year disease-free survival (DFS) was $98.5 \%$ and of five-year DFS was $89.1 \%$. Patients with early breast cancer had a one-year overall survival (OS) rate of $99.2 \%$ and a five-year OS rate of $95.6 \%$. The gradually decreased usage and shortened duration of tamixofen treatment, as well as reduced DFS and OS rates were observed in a 'real world'
\end{abstract}

Correspondence to: Dr Hong Zheng, Departments of Head and Neck and Mammary Gland Oncology, and Medical Oncology, Cancer Center; State Key Laboratory of Biotherapy, Laboratory of Molecular Diagnosis of Cancer, West China Hospital, Sichuan University, 37 Guo Xue Xiang, Chengdu, Sichuan 610041, P.R. China E-mail: hongzheng11@gmail.com

*Contributed equally

Key words: tamoxifen, early-stage breast cancer, application status, hormonal receptor-positive clinical setting. Improved treatment compliance in patients is recommended.

\section{Introduction}

Breast cancer is the most common cancer and is the leading cause of cancer mortality among females (1). In 2000, more than one million females were diagnosed with breast cancer and more than three million females succumbed to the disease (2). Tamoxifen as a selective estrogen receptor (ER) modulator is widely used in pre- and postmenopausal breast cancer (3). In 1977, tamoxifen was initially approved to be administered to cases of metastatic late breast cancer by the Food and Drug Administration. It has been utilized to reduce the recurrence and improve survival for ER-positive breast cancer, and to decrease the incidence of breast cancer in females $(4,5)$.

In previous years, it has been reported that endocrine therapy using tamoxifen reduced recurrence by $39 \%$ and the annual mortality rate by $31 \%$ (6). Endocrine therapy with adjuvant tamoxifen for five years also substantially reduces the rate of recurrence not only during the treatment period but also throughout the first decade (7). Inversely, no additional benefit from tamoxifen administered beyond five years has been demonstrated in females with ER-positive breast cancer and negative axillary lymph nodes (8). Despite this, strict criteria were used to select the patients in these clinical trials and characteristics varied in these patients. In fact, the majority of studies to establish the safety and efficacy of tamoxifen have been strict controlled and well-designed clinical studies, and few can challenge these studies. The reality of clinical trials, however, is that various factors may influence the study and lead to less-than-ideal results, for example, a lack of compliance in patients, which may affect the survival status of patients in certain ways.

Information management systems provide a new platform for 'real world' research. For example, Barron et al (9) reported that the cumulative tamoxifen nonpersistence rate was $22.1 \%$ within one year of commencing treatment with tamoxifen, and the cumulative nonpersistence rate by the end of follow-up at 3.5 years had increased to $35.2 \%$. Wigertz et al (10) revealed that $69 \%$ of patients were fully adherent to therapy for three years, and $12 \%$ prematurely discontinued therapy. A systematic review including 29 studies showed that $47.1 \%$ of patients 
discontinued therapy, which impacted the prognosis for breast cancer (11). To date, few studies exist on the therapy status of tamoxifen for Chinese patients with early breast cancer. Therefore, the aim of the present study was to investigate the therapy status of tamoxifen in patients with early hormonal receptor (HR)-positive breast cancer, to provide more information for clinical practice.

\section{Materials and methods}

Study design. This retrospective analysis was based on Chinese patients with early breast cancer for whom data was included in an information management system for breast cancer in the Huaxi Hospital of Sichuan University (Chengdu, China) between 1989 and 2012. The inclusion criteria of this study included: No distant metastasis during diagnosis with breast cancer; and a diagnosis of estrogen receptor and/or progesterone receptor (PR)-positive breast cancer, or an unclear HR status with tamoxifen used as the preferred drug. This study was approved by the institutional ethics committee of Huaxi Hospital.

Data source. The information management system for breast cancer in Huaxi Hospital covered information on diagnosis, surgery, chemotherapy, targeted therapy, endocrine therapy and radiotherapy. Information of patients with breast cancer was recorded, including general characteristics, history, diagnosis, auxiliary examination, therapy and living conditions. Real-time updates were recorded with regard to recurrence, metastasis condition and survival status. All patients received follow-up in outpatient clinics, or by telephone and email. It was ensured that there were follow-ups at least every 3-6 months within years 1-2 and at least every 6-12 months within years 3-5 following the diagnosis of breast cancer. When the updating information of patients was delayed more than once in the schedule, this was defined as a missing case.

Biomarker expression analysis. The expression of ER, PR and Ki-67 (an essential biomarker of cellular proliferation) in each patient was detected using immunohistochemical (IHC) analysis. The expression of ER and PR was recorded as negative or positive, and the percentages of ER and PR were calculated. Human epidermal growth factor receptor 2 (HER2) was assayed using the IHC method with the result presented as 0-3+. Negative HER2 was defined as 0 or $1+$, while positive HER2 was defined as $3+$. The samples with results of $2+$ and $1+$ for HER2 were then tested with a fluorescence in situ hybridization assay.

Disease-free survival (DFS) and overall survival (OS) analysis. The primary endpoint of this study was DFS, which was defined as the length of time from the date of diagnosis with breast cancer to the first recurrence (local, regional, distant metastasis or contralateral breast cancer), mortality from any cause or the last follow-up. Locoregional recurrence was defined as any recurrence of axillary fossa, internal mammary or supraclavicular lymph nodes in the homolateral breast or lymph drainage regions. The secondary endpoint was OS, which was defined as the length of time from the date of diagnosis with breast cancer to mortality from any cause or to the time of the last follow-up visit.
Statistical analysis. The information of all patients included in this study between 1989 and 2012 was exported from the information management system for breast cancer, and the deadline for follow-up was set to March 2013. The information was checked and organized by two researchers, and the statistical analysis was performed by two experienced researchers. The cumulative rates of continued tamoxifen treatment and survival time were visualized using Kaplan-Meier curves. All statistical analyses were performed using SPSS software, version 13.0 (SPSS, Inc., Chicago, IL, USA).

\section{Results}

Patient information. A total of 2,738 patients with HR-positive early breast cancer were included in this study based on the information management system. The general information and corresponding therapies of all patients are listed in Table I. The median age was 44 years, including $99.4 \%(2,721 / 2,738)$ female and $0.6 \%(17 / 2,738)$ male patients. Within females, $75.7 \%(2,059 / 2,738)$ were premenopausal when diagnosed with early breast cancer, $24.3 \%(661 / 2,738)$ were postmenopausal, and the median age of menopause was 47 years. During follow-up of female patients, $59.6 \%(1,624 / 2,738)$ were premenopausal, $36.3 \%(987 / 2,738)$ were postmenopausal and the menstruation status of the remaining $4.8 \%(130 / 2,738)$ could not be confirmed.

Pathological types and treatment of patients. Among all the patients, the main pathological carcinoma type was invasive ductal breast ( $85.1 \%$ ), followed by invasive (4.1\%), ductal in situ (DCIS, 3.3\%), mucinous (2.6\%) and invasive lobular (1.6\%). Tumor staging was based on clinical tumor node metastasis classification, and the rates of Tis/T0/T1/T2 and T3/T4 were $78.5 \%(2,150 / 2,738)$ and $9.4 \%(256 / 2,738)$, respectively. The regional node status of the patients was recorded, and the patients with a negative-regional node (N0) status accounted for $52.0 \%(1,423 / 2,738)$ of cases, while N1, N2 and N3 accounted for $28.5 \%$ (780/2,738), $10.8 \%(297 / 2,738)$ and $8.1 \%(222 / 2,738)$, respectively. A total of 374 patients $(13.7 \%)$ were ER-negative, and 2,063 patients $(75.4 \%)$ were ER-positive. For the PR, there were $14.0 \%$ negative cases and $74.5 \%$ positive cases. Patients with $\mathrm{Ki}-67$ values $\geq 14 \%$ accounted for $58.8 \%(1,611 / 2,738)$ of cases, while $13.8 \%(379 / 2,738)$ of patients exhibited values $<14 \%$. Following the IHC assay, $6.0 \%(165 / 2,738)$ of patients were shown to be HER2-positive.

Among the patients, $87.0 \%(2,380 / 2,738)$ of cases underwent modified radical mastectomy; 5.7\% (155/2,738), breast-conserving resections; $5.0 \%$ (139/2,738), extended radical mastectomy; and $2.3 \%(64 / 2,738)$, other surgeries. During follow-up, $40.0 \%(1,094 / 2,738), 6.5 \%(177 / 2,738)$ and $32.5 \%(891 / 2,738)$ of patients received anthracycline-, taxoland anthracycline plus taxol combination-based chemotherapy treatments, respectively. No chemotherapy was received by $7.8 \%(214 / 2,738)$ of patients.

Usage status of tamoxifen. Among the 7,234 patients included with early breast cancer amongst which the number of patients with HR-positive early breast cancer is 2,738. A total of $54.1 \%(3,913 / 7,234)$ of HR-positive and HR-unknown patients underwent more than one type of endocrine therapy, and the 
Table I. General information and corresponding therapy of patients included in the study.

\begin{tabular}{lcc}
\hline Patient information & Patients (n) & Percentage \\
\hline Total & 2738 & 100.0 \\
Female & 2721 & 99.4 \\
Male & 17 & 0.6
\end{tabular}

Menstruation when diagnosed

with early breast cancer ${ }^{\mathrm{a}}$

Postmenopause
Premenopause
Unknown

Menstruation during follow-up ${ }^{\mathrm{a}}$

Postmenopause
Premenopause
Unknown

Pathological carcinoma type

Ductal in situ

661

2059

1

Invasive ductal breast

Invasive

Mucinous

Invasive lobular

Others

T status

$\begin{array}{lr}\text { T0 } & 56 \\ \text { Tis } & 90 \\ \text { T1 } & 772 \\ \text { T2 } & 1232 \\ \text { T3 } & 183 \\ \text { T4 } & 73 \\ \text { TX } & 332\end{array}$

$\mathrm{N}$ status

$\begin{array}{lr}\text { N0 } & 1423 \\ \text { N1 } & 780 \\ \text { N2 } & 297 \\ \text { N3 } & 222 \\ \text { NX } & 16\end{array}$

Estrogen receptor

-
+
++
+++
\pm
Unknown

Progesterone receptor

-
+
++
+++
\pm

Table I. Continued.

Patient information Patients (n) Percentage

Progesterone receptor

Unknown

272

Human epidermal growth

factor receptor 2

\begin{tabular}{|c|c|c|}
\hline- & 1298 & 47.4 \\
\hline+ & 602 & 22.0 \\
\hline++ & 300 & 11.0 \\
\hline+++ & 165 & 6.0 \\
\hline \pm & 25 & 0.9 \\
\hline Unknown & 348 & 12.7 \\
\hline \multicolumn{3}{|l|}{ Ki-67 } \\
\hline$<14 \%$ & 379 & 13.8 \\
\hline$\geq 14 \%$ & 1611 & 58.8 \\
\hline Unknown & 748 & 27.3 \\
\hline \multicolumn{3}{|l|}{ WHO classification } \\
\hline I & 86 & 3.1 \\
\hline II & 576 & 21.0 \\
\hline III & 750 & 27.4 \\
\hline Unknown & 1326 & 48.4 \\
\hline \multicolumn{3}{|l|}{ Surgeries } \\
\hline Breast-conserving resections & 155 & 5.7 \\
\hline Modified radical mastectomy & 2380 & 87.0 \\
\hline Extended radical mastectomy & 139 & 5.0 \\
\hline Others & 64 & 2.3 \\
\hline \multicolumn{3}{|l|}{ Chemotherapy types } \\
\hline Anthracycline-based & 1094 & 40.0 \\
\hline Taxol-based & 177 & 6.5 \\
\hline Anthracycline plus taxol-based & 891 & 32.5 \\
\hline Others $^{b}$ & 362 & 13.2 \\
\hline None & 214 & 7.8 \\
\hline \multicolumn{3}{|l|}{ Adjuvant radiotherapy } \\
\hline Yes & 1004 & 36.7 \\
\hline No & 1734 & 63.3 \\
\hline
\end{tabular}

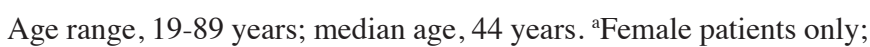
batients with unknown chemotherapy or missing data. WHO, World Health Organization; T, tumor; N, node.

preference of the other patients was tamoxifen treatment. The percentage of tamoxifen preference in adjuvant therapy was $97.3 \%$ prior to 2000 , and decreased to $60.4 \%$ in 2011 (Fig. 1). Among premenopausal patients, the percentage of tamoxifen preference reduced from $97.3 \%$ prior to 2000 to $89.5 \%$ in 2011 , while the percentage in postmenopausal patients declined from $97.4 \%$ prior to 2000 to $22.7 \%$ in 2011 (Fig. 2). Among patients receiving tamoxifen in sequence with aromatase inhibitors, those who used tamoxifen for one year accounted for $41.8 \%$. Subsequently, the percentages of patients using tamoxifen 


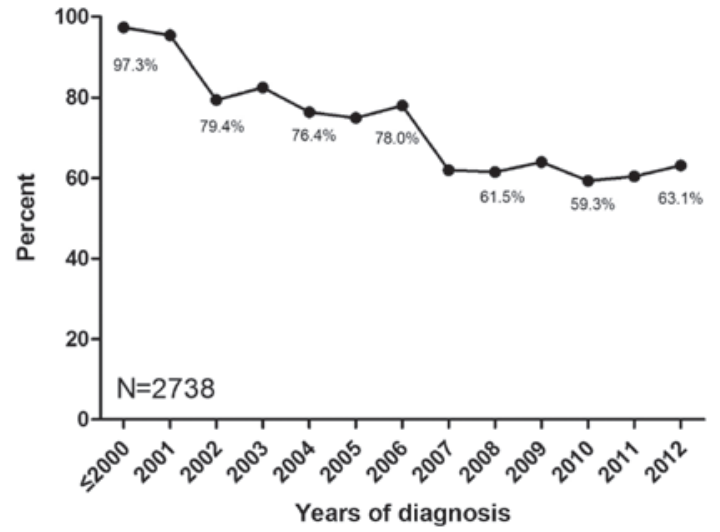

Figure 1. Percentage of tamoxifen preference in adjuvant endocrine therapy for early breast cancer. The horizontal axis represents the time period between 1989 and 2012, and the vertical axis represents the percentage of tamoxifen preference in adjuvant endocrine therapy. The patients who were newly diagnosed with early breast cancer in 2012 were undergoing adjuvant chemotherapy without endocrine therapy.

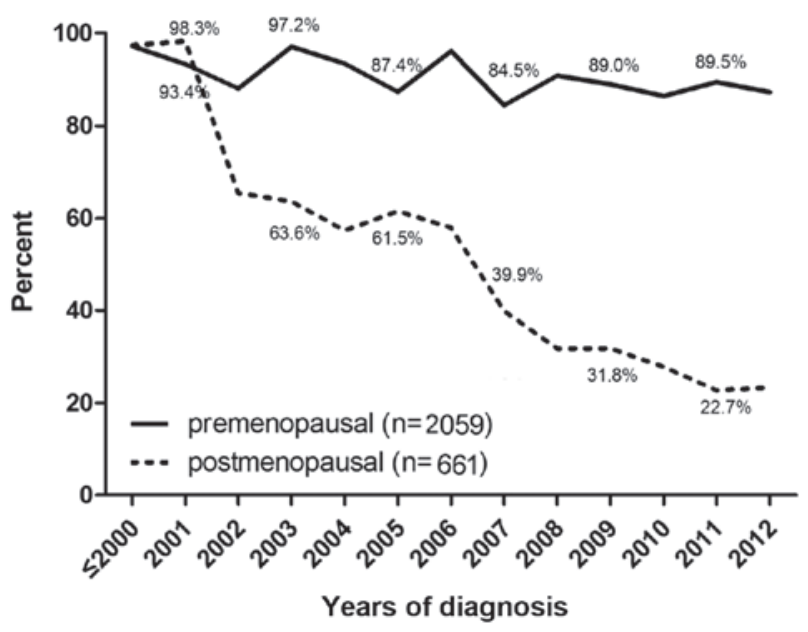

Figure 2. Percentage of tamoxifen treatment preference for early breast cancer in pre- and postmenopausal patients. The horizontal axis represents the time period between 1989 and 2012, and the vertical axis represents the percentage of tamoxifen preference in adjuvant endocrine therapy.

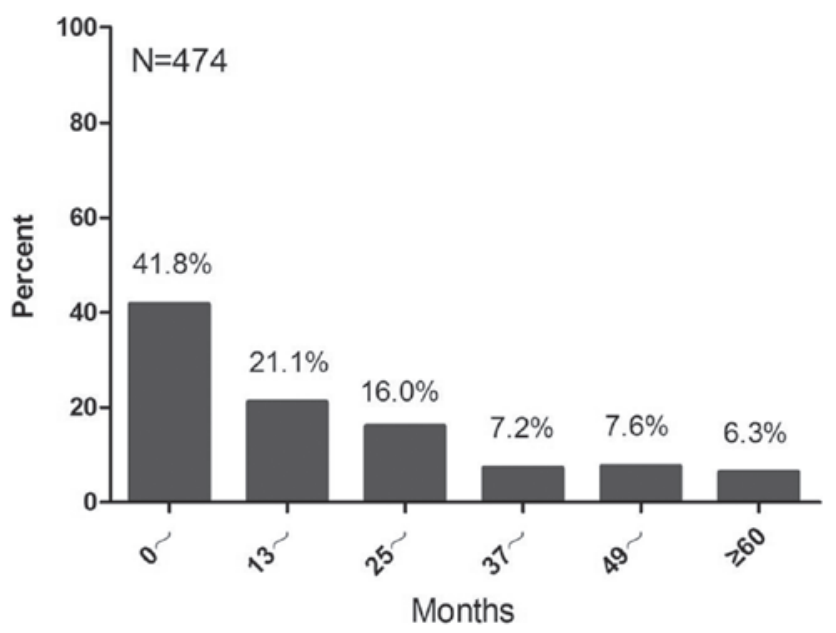

Figure 3. Status of tamoxifen in sequence with aromatase inhibitors in patients with early breast cancer $(n=474)$. The horizontal axis represents the time (months) and the vertical axis represents the percentage of tamoxifen use in sequence with aromatase inhibitors.

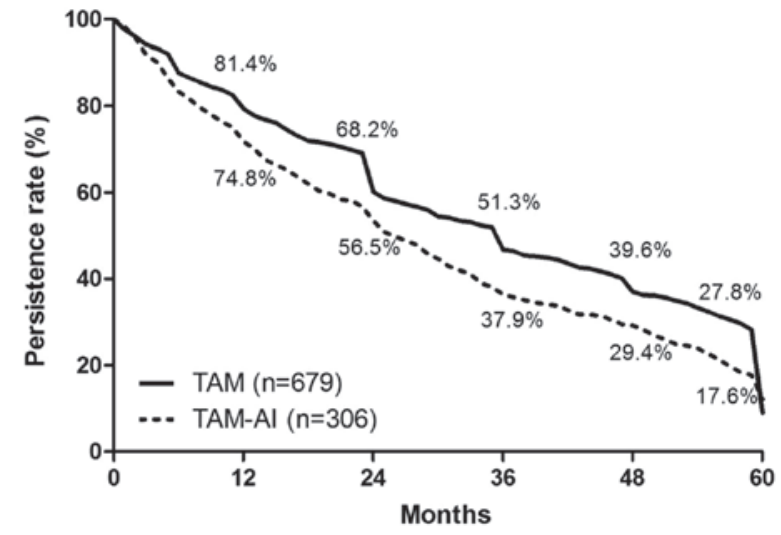

Figure 4. Kaplan-Meier curve analyzing the duration of TAM treatment preference and TAM treatment in sequence with AIs for early breast cancer. The horizontal axis represents the time (months) and the vertical axis represents the persistence rate. TAM, tamoxifen; AI, aromatase inhibitor.

A

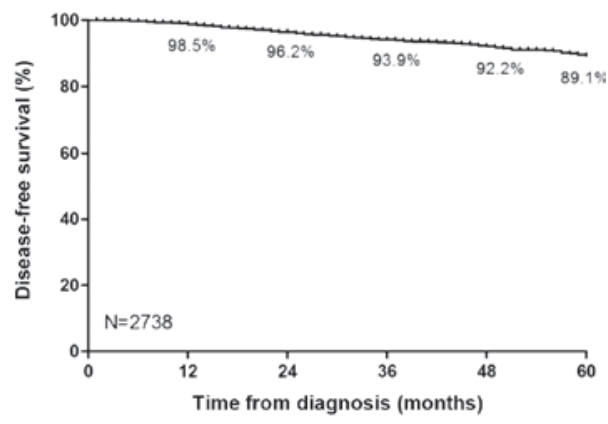

B

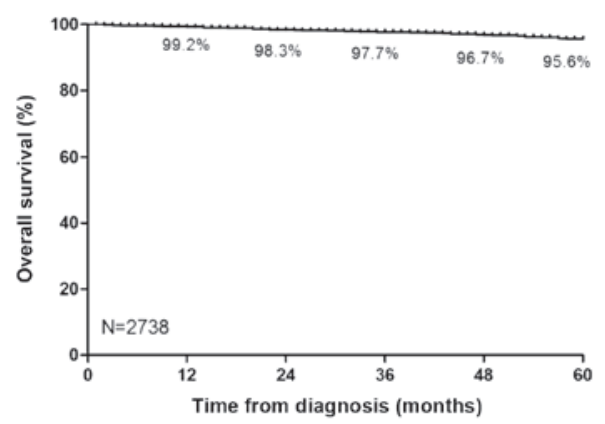

Figure 5. Kaplan-Meier curves analyzing the DFS and OS of patients with early breast cancer $(n=2,738)$. (A) The DFS and (B) OS of patients diagnosed with early breast cancer. The horizontal axis represents the time (months) and the vertical axis represents the corresponding rate. DFS, disease-free survival; OS, overall survial.

for $>2,3,4,6$ and 6 years were 21.1, 16.0, 7.2, 7.6 and $6.3 \%$, respectively (Fig. 3).

DFS and OS analysis of the patients. Follow-up statuses of the cases at the deadline revealed the following: $41.2 \%$ $(1,127 / 2,738)$ of cases were receiving endocrine therapy; $14.2 \%(388 / 2,738)$ were lost due to changes of the contact information; $2.3 \%(63 / 2,738)$ were deceased without updating information; $3.5 \%(97 / 2,738)$ lost contact; and $0.1 \%(2 / 2,738)$ refused follow-up. A duration analysis of the 985 patients $(36.0 \%)$ that ceased endocrine therapy was performed, and the duration of tamoxifen treatment for one, two, three, four and five years accounted for 79.4, 64.6, 47.1, 36.4 and 24.7\%, 
respectively (Fig. 4). With regard to the survival times of patients, the rate of one-year DFS was $98.5 \%$, and the DFS rates for two, three, four and five years were 96.2, 93.9, 92.2 and $89.1 \%$, respectively (Fig. 5A). Patients with early breast cancer had a one-year OS rate of $99.2 \%$, and the rates of OS for two, three, four and five years were 98.3, 97.7, 96.7 and $95.6 \%$, respectively (Fig. 5B).

\section{Discussion}

'Real world' studies are a method of evaluating the external validity and safety of real therapy, based on a larger sample covering a representative population (12-14). In the present study, according to their actual conditions and own free will, all patients were non-randomly selected for treatment, and an evaluation of long-term adjuvant therapy with tamoxifen was performed. To date, randomized controlled trials (RCTs) remain the gold standard of studies (15). However, strict inclusion standards do not conform to the characteristics of patients in clinical practice, and whether the results can be directly applied to clinical settings is uncertain. Additionally, sample size determines the effectiveness of corresponding studies, and the lower incidence is hard to verify in RCTs. Notably, it may not be possible to perform an RCT due to the relevant ethics and legal regulations $(12,16)$.

Therefore, information management systems provide new insights into further studies in the 'real world'. Integrated studies combining prospective with retrospective methods have been used to collect information, which overcomes the selective migration in retrospective studies, and applies a maximum of intent-to-treat analysis to ensure more comprehensive data and real results $(17,18)$. In the present study, the application status of tamoxifen in patients with early breast cancer was investigated based on data in an information management system. It was revealed that the usage of tamoxifen decreased gradually over time, and the duration of tamoxifen treatment also reduced as well as the DFS and OS of patients.

Tamoxifen, as the foundation of endocrine therapy for breast cancer, plays a crucial role in treating patients prior to menopause. It has been reported that the optimum duration of tamoxifen treatment is five years (19). It is controversial as to whether benefits arise from prolonging the treatment time. Recently, adjuvant therapy with tamoxifen for 10 years was found to reduce relapse $(617 / 3,428$ vs. $711 / 3,418, \mathrm{P}=0.002)$ and mortality (331 vs. $397, \mathrm{P}=0.01$ ) when compared with adjuvant therapy with tamoxifen for five years (7). In the present study, it was revealed that the patients who continued tamoxifen treatment for one, two, three, four and five years accounted for $79.4,64.6,47.1,36.4$ and $24.7 \%$ of cases, respectively. The usage of long-term endocrine therapy was lower than that in another retrospective study by Hershman et al (20), which indicated $49 \%$ usage for 4.5 years, and was also lower than the $72-90 \%$ usage shown in other previous RCTs (21-23). Additionally, our previous study showed that $>10 \%$ of HR-positive patients did not receive any endocrine therapy every year (unpublished data). Furthermore, the DFS and OS differed significantly between patients with and without endocrine therapy (five year DFS: 88.4 vs.75.1\%; five year OS: 95.7 vs. $83.8 \%$ ), which was consistent with the study by Hershman et al (20). Overall, in the 'real world', certain patients did not continue to receive endocrine therapy, and it is recommended that treatment compliance in patients should be improved to prolong the survival time of patients with breast cancer.

In the findings of the Arimidex ${ }^{\circledR}$, Tamoxifen, Alone or in Combination (ATAC) randomized trial in 2002, during adjuvant endocrine therapy for early breast cancer, the three-year DFS rate for anastrozole use was improved compared with that for tamoxifen [89.4 vs. 87.4\%; hazard ratio, 0.83 ; $95 \%$ confidence interval $(\mathrm{CI}), 0.71-0.96 ; \mathrm{P}=0.013]$, and subgroup analysis revealed increased benefits in HR-positive patients (24). However, anastrozole is only considered for use in patients for whom tamoxifen is contraindicated or not tolerated (25). A study on postmenopausal women with early breast cancer in 2005 showed that adjuvant treatment with letrozole reduced the risk of recurrent disease to a greater extent than tamoxifen as indicated by the five-year DFS rates $(84.0 \%$ vs. $81.4 \%$; hazard ratio, 0.81 ; 95\% CI, 0.70-0.93; $\mathrm{P}=0.003)$, particularly at distant sites (26). The St Gallen International Expert Consensus in 2009 recommend that aromatase inhibitors should form part of the standard endocrine therapy for postmenopausal women with HR-positive breast cancer (27). Thus, these observations challenge the application of tamoxifen in postmenopausal women with breast cancer, and the results of the present study reflect that the status of tamoxifen has gradually wavered. With increasing evidence of the efficacy of aromatase inhibitors, the usage of tamoxifen was revealed by the present study to be only $19.7 \%$ in 2011 . Notably, either adjuvant tamoxifen or an aromatase inhibitor is the first choice in treating postmenopausal women with early breast cancer, not due to the survival advantage, but for the long-term efficacy and reduced recurrence rate $(26,28)$.

In conclusion, adjuvant tamoxifen as a treatment for early breast cancer has been surveyed in this study in a 'real world' context, and the results showed that the usage of tamoxifen decreased gradually and the duration of use was reduced over time, as well as the DFS and OS of patients being reduced to some extent. Additionally, this result suggests that the treatment compliance in patients was poor. The use of tamoxifen for one year in sequence with aromatase inhibitors accounted for $41.8 \%$ of cases, and whether this treatment affects the survival of patients requires further study. It is anticipated that these results may provide new insights into studies in a 'real world' context.

\section{References}

1. Althuis MD, Dozier JM, Anderson WF, Devesa SS and Brinton LA: Global trends in breast cancer incidence and mortality 1973-1997. Int J Epidemiol 34: 405-412, 2005.

2. Parkin DM Bray FI and Devesa SS: Cancer burden in the year 2000. The global picture. Eur J Cancer 37 (Suppl 8): S4-S66, 2001.

3. Park WC and Jordan VC: Selective estrogen receptor modulators (SERMS) and their roles in breast cancer prevention. Trends Mol Med 8: 82-88, 2002.

4. Cohen MH, Hirschfeld S, Flamm Honig S, et al: Drug approval summaries: arsenic trioxide, tamoxifen citrate, anastrazole, paclitaxel, bexarotene. Oncologist 6: 4-11, 2001.

5. Fisher B, Costantino JP, Wickerham DL, et al: Tamoxifen for prevention of breast cancer: report of the National Surgical Adjuvant Breast and Bowel Project P-1 Study. J Natl Cancer Inst 90: 1371-1388, 1998. 
6. Early Breast Cancer Trialists' Collaborative Group (EBCTCG): Effects of chemotherapy and hormonal therapy for early breast cancer on recurrence and 15-year survival: an overview of the randomised trials. Lancet 365: 1687-1717, 2005.

7. Davies C, Pan H, Godwin J, et al; Longer Against Shorter (ATLAS) Collaborative Group: Long-term effects of continuing adjuvant tamoxifen to 10 years versus stopping at 5 years after diagnosis of oestrogen receptor-positive breast cancer: ATLAS, a randomised trial. Lancet 381: 805-816, 2013.

8. Fisher B, Dignam J, Bryant J and Wolmark N: Five versus more than five years of tamoxifen for lymph node-negative breast cancer: updated findings from the National Surgical Adjuvant Breast and Bowel Project B-14 randomized trial. J Natl Cancer Inst 93: 684-690, 2001.

9. Barron TI, Connolly R, Bennett K, Feely J and Kennedy MJ: Early discontinuation of tamoxifen: a lesson for oncologists. Cancer 109: 832-839, 2007.

10. Wigertz A, Ahlgren J, Holmqvist M, et al: Adherence and discontinuation of adjuvant hormonal therapy in breast cancer patients: a population-based study. Breast Cancer Res Treat 133 367-373, 2012.

11. Murphy CC, Bartholomew LK, Carpentier MY, Bluethmann SM and Vernon SW: Adherence to adjuvant hormonal therapy among breast cancer survivors in clinical practice: a systematic review. Breast Cancer Res Treat 134: 459-478, 2012.

12. Thorpe KE, Zwarenstein M, Oxman AD, et al: A pragmatic-explanatory continuum indicator summary (PRECIS): a tool to help trial designers. J Clin Epidemiol 62: 464-475, 2009.

13. Booth CM and Mackillop WJ: Translating new medical therapies into societal benefit: the role of population-based outcome studies. JAMA 300: 2177-2179, 2008.

14. Krumholz HM: Real-world imperative of outcomes research. JAMA 306: 754-755, 2011

15. Kaptchuk TJ: The double-blind, randomized, placebo-controlled trial: gold standard or golden calf? J Clin Epidemiol 54: 541-549, 2001.

16. Hershman DL and Wright JD: Comparative effectiveness research in oncology methodology: observational data. J Clin Oncol 30: 4215-4222, 2012

17. Trotter JP: Patient registries: a new gold standard for 'real world' research. Ochsner J 4: 211-214, 2002.

18. Parkin DM: The evolution of the population-based cancer registry. Nat Rev Cancer 6: 603-612, 2006.
19. Albain KS, Barlow WE, Ravdin PM, etal; Breast CancerIntergroup of North America: Adjuvant chemotherapy and timing of tamoxifen in postmenopausal patients with endocrine-responsive, node-positive breast cancer: a phase 3, open-label, randomised controlled trial. Lancet 374: 2055-2063, 2009.

20. Hershman DL, Shao T, Kushi LH, et al: Early discontinuation and non-adherence to adjuvant hormonal therapy are associated with increased mortality in women with breast cancer. Breast Cancer Res Treat 126: 529-537, 2011.

21. Goss PE, Ingle JN, Martino S, et al: A randomized trial of letrozole in postmenopausal women after five years of tamoxifen therapy for early-stage breast cancer. N Engl J Med 349: 1793-1802, 2003

22. Coombes RC, Hall E, Gibson LJ, et al; Intergroup Exemestane Study: A randomized trial of exemestane after two to three years of tamoxifen therapy in postmenopausal women with primary breast cancer. N Engl J Med 350: 1081-1092, 2004.

23. Howell A, Cuzick J, Baum M, et al; ATAC Trialists' Group: Results of the ATAC (Arimidex, Tamoxifen, Alone or in Combination) trial after completion of 5 years' adjuvant treatment for breast cancer. Lancet 365: 60-62, 2005.

24. Baum M, Budzar A, Cuzick J, et al; ATAC Trialists' Group: Anastrozole alone or in combination with tamoxifen versus tamoxifen alone for adjuvant treatment of postmenopausal women with early breast cancer: first results of the ATAC randomised trial. Lancet 359: 2131-2139, 2002

25. Goldhirsch A, Wood WC, Gelber RD, et al: Meeting highlights: updated international expert consensus on the primary therapy of early breast cancer. J Clin Oncol 21: 3357-3365, 2003.

26. Thürlimann B, Keshaviah A, Coates AS et al; Breast International Group (BIG) 1-98 Collaborative Group: A comparison of letrozole and tamoxifen in postmenopausal women with early breast cancer. N Engl J Med 353: 2747-2757, 2005.

27. Goldhirsch A, Ingle JN, Gelber RD, et al; Panel members: Thresholds for therapies: highlights of the St Gallen International Expert Consensus on the primary therapy of early breast cancer 2009. Ann Oncol 20: 1319-1329, 2009.

28. Forbes JF, Cuzick J, Buzdar A, et al; Arimidex, Tamoxifen, Alone or in Combination (ATAC) Trialists' Group: Effect of anastrozole and tamoxifen as adjuvant treatment for early-stage breast cancer: 100-month analysis of the ATAC trial. Lancet Oncol 9: 45-53, 2008. 